\title{
Accurate multicomponent Fick diffusion at a lower cost than mixture-averaged approximation: validation in steady and unsteady counterflow flamelets
}

\author{
Bertrand Naud ${ }^{*, a}$, Manuel Arias-Zugasti ${ }^{\mathrm{b}}$ \\ ${ }^{a}$ Modelling and Numerical Simulation Group, Energy Department, CIEMAT, Avda. Complutense 22, 28040 Madrid, Spain \\ ${ }^{b}$ Departamento de Física Matemática y de Fluidos, Facultad de Ciencias UNED, Senda del Rey 9, 28040 Madrid, Spain
}

\begin{abstract}
Neglecting the effect of thermal diffusion (Soret effect), we consider different formulations of multicomponent diffusion as proposed by Arias-Zugasti et al., Combust. Flame 163:540-556 (2016), for mixtures of dilute gases with large numbers of components. In particular, we detail the practical implementation of Model $1+M$ (loc.cit.) using the lowest order approximation. This is a simple and easy to implement approach, where the $1+M$ main species can be chosen locally. These new formulations of multicomponent diffusion are compared to the formulation of Dixon-Lewis, used for instance in the Chemkin package, and also to the widely used mixture-averaged simplification. Steady flamelets are first considered for very different fuels (hydrogen, methane or dodecane) in order to show some differences and limitations of the different formulations, and in order to compare computational costs when different numbers of species are involved. An unsteady auto-igniting counterflow diffusion flamelet of methane in a coflow of hot products is also considered. In this way, unsteady $1 \mathrm{D}$ calculations can be performed, still including all the challenges of multicomponent diffusion transport as would appear for instance in Direct Numerical Simulations (DNS) of turbulent flames. The different comparisons in terms of precision and cost show that Model $1+M$ truncated to the lowest order can be more efficient than the mixture-averaged approach, while reproducing the results of Dixon-Lewis multicomponent diffusion. The efficiency of the proposed approach is mainly due to the evaluation of fewer binary diffusion coefficients, therefore reducing significantly the number of time-consuming operations. Finally, we show that the definition of $1+M$ main species can also be used to simplify the time-consuming evaluation of the mixture viscosity, leading to an important further reduction of CPU time that makes the lowest order Model $1+M$ always more efficient than the mixture-averaged formulation.
\end{abstract}

Key words: Differential diffusion, multicomponent gases, mixture-averaged

\section{Introduction}

In order to accurately represent flames in numerical simulations, the terms in the convection-diffusion-reaction problem need to include all the relevant physical processes. Direct $\mathrm{Nu}-$ merical Simulations (DNS) of laminar or turbulent flames can then be considered as numerical experiments and provide complete data sets allowing to understand in detail the combustion processes. In particular, combustion may involve some fuel components or radicals (like $\mathrm{H}_{2}$ or $\mathrm{H}$ ) that diffuse much more rapidly than larger molecules (like $\mathrm{O}_{2}, \mathrm{~N}_{2}, \mathrm{H}_{2} \mathrm{O}, \mathrm{CH}_{4}, \mathrm{CO}_{2}$, $\mathrm{CO}, \ldots)$. In these cases, and in particular when diffusion of species play a key role like in non-premixed combustion, an accurate numerical simulation should include a correct approach to treat the species diffusion fluxes.

In the case of mixtures of dilute gases, the Kinetic Theory of Gases provides the general framework for the calculation of the molecular transport coefficients of the mixture [2, 3, 4]. In this regard, a detailed approach is the multicomponent diffusion formulation of Dixon-Lewis [5]. In this approach, in order to calculate the muticomponent diffusion matrix a $N \times N$ matrix

\footnotetext{
${ }^{*}$ Corresponding author

Email address: bertrand.naud@ciemat. es (Bertrand Naud)
}

needs to be build and inverted, where $N$ is the number of species in the mixture. Moreover, $N^{2}$ binary diffusion coefficients $\mathcal{D}_{i j}$ (diffusion of species $i$ in species $j$ ) need to be evaluated. One way to evaluate the binary diffusion coefficients (and other pure species properties, as viscosity and thermal conductivity) is the method of Monchick and Mason [6, 7]. In this paper, instead of these complex expressions we use polynomial fits in order to evaluate pure-species properties in the same way as done in Chemkin [7].

In the case of mixtures with large numbers of components, a widely used simplification is the so-called mixture-averaged diffusion model [8], where the $N^{2}$ binary diffusion coefficients $\mathcal{D}_{i j}$ are required, but where no matrix needs to be inverted. The results are often quite close to the Dixon-Lewis approach, but they may imply relevant differences in some cases involving very diffusive species (like $\mathrm{H}_{2}$ ).

Following the idea that in multicomponent mixtures, and in particular in combustion, only a small subset of major species is relevant while the other species are dilute, and extending the work of [9], Arias-Zugasti et al. [1] recently presented a power series expansion approximation for multicomponent diffusion (truncated to a given order) termed as "Model 1" (that can be compared to the iteration procedure for diffusion velocities introduced by Jones and Boris [10] and Oran and Boris 
[11], and the posterior works of Giovangigli, as for instance $[12,13])$. Moreover, Arias-Zugasti et al. [1] also introduced a so-called "Model $1+M$ ", where the previous approximation is only applied to the most diluted species (a similar idea is used by Xin et al. [14], where they consider some "criticaldiffusivity-species"). The truncated power series expansion Model 1 avoids to invert the $N \times N$ matrix. On the other hand, Model $1+M$ only implies the inversion of a small $M \times M$ matrix, where $1+M$ is the number of non-diluted species.

In this paper, we consider Model $1+M$ using the lowest order approximation (i.e., truncated at order zero: termed as Model $\left.[1+M]^{(0)}\right)$. We show that keeping enough main species ( $M$ large enough), this is the most efficient way to implement Model $1+M$. We show how Model $[1+M]^{(0)}$ compares to the formulation of Dixon-Lewis, and we quantify the gain in computational time. We choose to consider simple 1D configurations of counterflow diffusion flames, containing most of the issues that would have to be dealt with in a realistic DNS.

\section{Multicomponent diffusion formulations}

\subsection{Dixon-Lewis formulation}

Neglecting the effect of thermal diffusion, the multicomponent species flux can be written using the notation of Ferziger and Kaper [4]:

$$
\rho Y_{i} \mathbf{V}_{i}=-\rho Y_{i} \sum_{j=1}^{N} D_{i j} \mathbf{d}_{j}=-\rho Y_{i} \sum_{j=1}^{N-1}\left(D_{i j}-D_{i N}\right) \mathbf{d}_{j}
$$

where $\rho$ is the mixture density, $Y_{i}$ are the species mass fraction, $\mathbf{V}_{i}$ the diffusion velocity vectors for species $i$ and $D_{i j}$ are the multicomponent diffusion coefficients. In its simplest form, the diffusion driving force vector can be simply expressed as the gradient of the mole fraction $X_{i}$, such that $\mathbf{d}_{i}=\nabla X_{i}$. Since the sum of species fluxes should be zero, we can also express the flux as a sum over $N-1$ species, as in the second relation in the above equation. As shown in [1], in order to calculate the multicomponent diffusion coefficients $D_{i j}$ it is convenient to introduce the dimensionless multicomponent diffusion factors $c_{i j}$, defined by:

$$
c_{i j}=X_{i} \frac{D_{i j}-D_{i N}}{\mathcal{D}_{i N}}
$$

where $\mathcal{D}_{i j}$ are the binary diffusion coefficients (evaluated here using polynomial fits [7]). Equation (1) can then be re-written as:

$$
\rho Y_{i} \mathbf{V}_{i}=-\rho \frac{W_{i}}{\bar{W}} \mathcal{D}_{i N} \sum_{j=1}^{N-1} c_{i j} \nabla X_{j}
$$

with $W_{i}$ the molecular mass of species $i$ and $\bar{W}$ the mean molecular mass of the mixture. Thus, in [1] it is shown that according to the Kinetic Theory of Gases, the problem of computing the multicomponent diffusion matrix $D_{i j}$ reduces to inverting the matrix $(\mathbb{1}+A)_{i j}$ :

$$
c_{i j}=(\mathbb{1}+A)_{i j}^{-1}
$$

where matrix $A$ is given by:

$$
A_{i j}=\sum_{k=1}^{N-1} X_{k}\left(\frac{\mathcal{D}_{i N}}{\mathcal{D}_{i k}}-1\right) \delta_{i j}+X_{i}\left(\frac{W_{j}}{W_{N}}-\frac{\mathcal{D}_{i N}}{\mathcal{D}_{i j}}\right) \frac{\mathcal{D}_{j N}}{\mathcal{D}_{i N}}
$$

It is useful to express the above relation in a less compact form in order to show that the binary coefficients $\mathcal{D}_{i i}$ are not required:

$$
\begin{aligned}
& A_{i i}=\sum_{k=1, k \neq i}^{N-1} X_{k}\left(\frac{\mathcal{D}_{i N}}{\mathcal{D}_{i k}}-1\right)+X_{i}\left(\frac{W_{i}}{W_{N}}-1\right) \\
& A_{i j}=X_{i}\left(\frac{W_{j} \mathcal{D}_{j N}}{W_{N} \mathcal{D}_{i N}}-\frac{\mathcal{D}_{j N}}{\mathcal{D}_{i j}}\right), \quad i \neq j
\end{aligned}
$$

Equations (1)-(5) correspond to the standard Dixon-Lewis formulation, when the $(N-1) \times(N-1)$ matrix $(\mathbb{1}+A)_{i j}$ is directly inverted.

\subsection{Truncated power series expansion models (Model 1)}

As detailed by Arias-Zugasti et al. [1], Eq. (4) can be formally expanded as a Von-Neumann series, thus providing the power series expansion solution termed as Model 1. The first order truncation of the power series expansion leads to the following expression (Model 1 with first order corrections):

$$
c_{i j}^{(1)}=\delta_{i j}-A_{i j}
$$

and the second order truncation (Model 1 with second order corrections) reads:

$$
c_{i j}^{(2)}=\delta_{i j}-A_{i j}+\sum_{k=1}^{N-1} A_{i k} A_{k j}
$$

while the lowest order approximation would be $c_{i j}^{(0)}=\delta_{i j}$, where $\delta_{i j}$ is the Kronecker delta.

\subsection{Lowest order Model $1+M\left(\right.$ Model $\left.[1+M]^{(0)}\right)$}

The model is based on the observation that even though Model 1 has slow convergence rate if the mixture departs from the dilute limit $\left(X_{i} \ll 1, i=1, \ldots, N-1\right)$, low order approximations are valid for diluted species. Therefore, the idea is to define the $1+M$ main species locally (as the species with largest concentrations) and use no approximation for these main species, while Model 1 truncated at the lowest order is used for species with low concentrations.

Hence, the reference species $K$ is chosen to be the species with the largest concentration, and we identify the other main species $i$ satisfying:

$$
X_{i} \geq \gamma \cdot X_{K}
$$

where $\gamma$ is a threshold factor. We reorder the species such that $K=N$ and that the other $M$ main species satisfying (10) are the first species in the list $(1 \leq i \leq M)$.

In this way, we can directly apply the lowest order approximation proposed by Arias-Zugasti et al. [1]:

$$
c_{i j}^{[1+M]^{(0)}}=\left(\begin{array}{c|c}
{\left[\mathbb{1}+A_{11}^{(0)}\right]^{-1}} & -\left[\mathbb{1}+A_{11}^{(0)}\right]^{-1} \cdot A_{12}^{(0)} \cdot\left[\mathbb{1}+A_{22}^{(0)}\right]^{-1} \\
\hline 0 & {\left[\mathbb{1}+A_{22}^{(0)}\right]^{-1}}
\end{array}\right)
$$


In this approach we need to invert the $M \times M$ matrix $\left(\mathbb{1}+A_{11}^{(0)}\right)$ where $A_{11}^{(0)}$ is defined using Eq. (5) considering only $M$ main species:

$$
\begin{aligned}
& 1 \leq i, j \leq M \\
& \left(A_{11}^{(0)}\right)_{i j}=\sum_{k=1}^{M} X_{k}\left(\frac{\mathcal{D}_{i N}}{\mathcal{D}_{i k}}-1\right) \delta_{i j}+X_{i}\left(\frac{W_{j}}{W_{N}}-\frac{\mathcal{D}_{i N}}{\mathcal{D}_{i j}}\right) \frac{\mathcal{D}_{j N}}{\mathcal{D}_{i N}}
\end{aligned}
$$

On the other hand the $M \times(N-1-M)$ matrix $A_{12}^{(0)}$ is defined as:

$$
\begin{aligned}
& 1 \leq i \leq M \text { and } M+1 \leq j \leq N-1, \\
& \left(A_{12}^{(0)}\right)_{i j}=X_{i}\left(\frac{W_{j}}{W_{N}}-\frac{\mathcal{D}_{i N}}{\mathcal{D}_{i j}}\right) \frac{\mathcal{D}_{j N}}{\mathcal{D}_{i N}}
\end{aligned}
$$

Finally, the $(N-1-M) \times(N-1-M)$ matrix $A_{22}^{(0)}$ is diagonal, such that it is straightforward to obtain the inverse matrix $\left[\mathbb{1}+A_{22}^{(0)}\right]^{-1}$ :

$$
\begin{aligned}
& M+1 \leq i, j \leq N-1 \\
& {\left[\mathbb{1}+A_{22}^{(0)}\right]_{i j}^{-1}=\delta_{i j} /\left[1+\sum_{k=1}^{M} X_{k}\left(\frac{\mathcal{D}_{i N}}{\mathcal{D}_{i k}}-1\right)\right]}
\end{aligned}
$$

Summarising, we find that in this case (3) can be simplified for the diluted species, leading to:

$$
\begin{array}{lr}
i \leq M, & \rho Y_{i} \mathbf{V}_{i}=-\rho \frac{W_{i}}{\bar{W}} \mathcal{D}_{i N} \sum_{j=1}^{N-1} c_{i j} \nabla X_{j} \\
M<i<N, & \rho Y_{i} \mathbf{V}_{i}=-\rho \frac{W_{i}}{\bar{W}} \mathcal{D}_{i N} c_{i i} \nabla X_{i} \\
i=N, & \rho Y_{N} \mathbf{V}_{N}=-\sum_{j=1}^{N-1} \rho Y_{j} \mathbf{V}_{j}
\end{array}
$$

\subsection{Mixture-averaged model}

We are also interested in comparing these multicomponent diffusion formulations to the so-called mixture-averaged diffusion model [8]. This simplified expression consists in evaluating an effective diffusion coefficient $D_{i}$ of the $i$-th species into the mixture as:

$$
D_{i}=\frac{1-Y_{i}}{\sum_{j \neq i} X_{j} / \mathcal{D}_{j i}}
$$

leading to the following simple expression for the multicomponent species fluxes (here neglecting the effect of thermal diffusion):

$$
\rho Y_{i} \mathbf{V}_{i}=-\rho \frac{W_{i}}{\bar{W}} D_{i} \nabla X_{i}
$$

In this case, a correction velocity $\mathbf{V}_{c}$ is added to the species diffusive velocity $\mathbf{V}_{i}$ in order to ensure that the net species diffusion flux is zero:

$$
\mathbf{V}_{c}=\sum_{j=1}^{N} Y_{i} \mathbf{V}_{i}
$$

2.5. Correspondence and differences with Xin et al. reduced multicomponent diffusion model

The purpose of the current paper is not to consider a more efficient model based on the mixture-averaged model. However, the definition of the $1+M$ species using the threshold factor $\gamma$ given by (10) could be used to reduce the sum in (16), as desbribed in Appendix B. In this case, a more efficient model can be obtained by reducing the number of binary diffusion coefficients to be evaluated. This possible reduced mixture-averaged model is interesting in order to better understand the modelling of the diluted species $M<i<N$ in Model $[1+M]^{(0)}$ on the second line in (15), since we can verify that it corresponds to (16), where $Y_{i}$ is neglected and where the sum is over the $1+M$ main species only.

In this sense, we verify that the $1+M$ model of Arias-Zugasti et al. [1] reduces in its lowest order approximation to a similar model as the reduced multicomponent diffusion model proposed by Xin et al. [14]. The main improvement of the model proposed here is twofold: the definition of the $1+M$ main species (the "critical-diffusivity-species" considered by Xin et $a l$.) is local, and not all the binary diffusion coefficients need to be evaluated since a reduced mixture-averaged model is used for the diluted species.

\subsection{Computational cost}

In Table 1, for each multicomponent diffusion approach, we summarise the number of binary coefficients $\mathcal{D}_{i j}$ to be computed, the required number of multiplications and divisions when evaluating the matrix components $A_{i j}$ or the mixtureaveraged diffusion coefficients $D_{i}$, and the size of the matrix to be inverted (together with the order of number of operations involved in the evaluation of $c_{i j}$ ). We also indicate the number of multiplications in Eq. (1), (3), (15) and (17), considering that $\rho / \bar{W}$ and $\left(\mathcal{D}_{i N} \cdot c_{i j}\right)$ are given pre-calculated coefficients. The small additional cost related to the correction velocity in the mixture-averaged implementation is not considered here.

In a standard $N \times N$ matrix implementation of the DixonLewis formulation, we need to evalute $N(N-1) / 2$ binary diffusion coefficients $\mathcal{D}_{i j}$ (since the $N$ coefficients $\mathcal{D}_{i i}$ are not needed and the other $N(N-1) / 2$ coefficients are obtained as $\mathcal{D}_{j i}=\mathcal{D}_{i j}$ ) and the $N^{2}$ components of a $N \times N$ matrix, moreover, we need to invert the $N \times N$ matrix. The mixture-averaged formulation only requires the evaluation of $N(N-1) / 2$ binary diffusion coefficients $\mathcal{D}_{i j}$ and $N$ effective diffusion coefficients $D_{i}$. With Model 1 , we need to evaluate $N(N-1) / 2$ binary diffusion coefficients, and we need to evaluate $(N-1)^{2}$ components of $A_{i j}$, but no matrix needs to be inverted since Eq. (8) or (9) are used instead. In the Model $1+M$ at lowest order, we can see that we only need $M(M-1) / 2+M(N-1-M)$ binary coefficients, since we need to evaluate $M(M-1) / 2$ coefficients in (12) and the same $M(N-1-M)$ coefficients $\mathcal{D}_{i j}$ or $\mathcal{D}_{j i}$ in (13) or (14). We need to compute the $(N-1)(1+M)-M$ components of the matrices $A_{11}^{(0)}, A_{12}^{(0)}$ and $A_{22}^{(0)}$. In this case, only a small $M \times M$ matrix needs to be inverted. The value of $M$ depends on the value of the threshold factor $\gamma$ used to identify the main species in (10). 
Table 1: Estimation of computational cost of different multicomponent diffusion approaches: number of coefficients $\mathcal{D}_{i j}$ to be computed (taking advantage of the symmetry $\mathcal{D}_{i j}=\mathcal{D}_{j i}$ ), number of multiplications and divisions in matrix coefficient evaluation, size of matrix to be inverted ( $\rightarrow$ order of number of operations involved) and number of multiplications in final evaluation of the fluxes.

\begin{tabular}{|c|c|c|c|c|c|}
\hline & & Dixon-Lewis & Mix.-av. & Model 1 & Model $[1+M]^{(0)}$ \\
\hline $\mathcal{D}_{i j}$ & & $N(N-1) / 2$ & $N(N-1) / 2$ & $N(N-1) / 2$ & $M(M-1) / 2+M(N-1-M)$ \\
\hline$A_{i j}\left(\right.$ or $\left.D_{i}\right)$ & & Eq. (5) & Eq. (16) & Eq. (5) & Eq. (12), (13) and (14) \\
\hline & mult. & $3 N^{2}$ & - & $3(N-1)^{2}$ & $3 M(N-1)$ \\
\hline & div. & $4 N^{2}$ & $N^{2}$ & $4(N-1)^{2}$ & $4 M(N-1)$ \\
\hline$c_{i j}$ & {$[\mathbb{1}+A]^{-1}$} & $\begin{array}{c}N \times N \text { inverse matrix } \\
\rightarrow O\left(N^{3}\right)\end{array}$ & - & $\begin{array}{l}\text { Eq. (8) or Eq. (9) } \\
\rightarrow O\left(N^{2}\right) \text { or } O\left(N^{3}\right)\end{array}$ & $\begin{array}{c}M \times M \text { inverse matrix } \\
\rightarrow O\left(M^{3}\right)\end{array}$ \\
\hline fluxes & mult. & $\begin{array}{c}\text { Eq. (1) } \\
N(N+1)\end{array}$ & $\begin{array}{l}\text { Eq. (17) } \\
3 N\end{array}$ & $\begin{array}{c}\text { Eq. (3) } \\
(N-1)(N+1)\end{array}$ & $\begin{array}{c}\text { Eq. (15) } \\
M(N+1)+3(N-1-M)\end{array}$ \\
\hline
\end{tabular}

Table 1 already indicates that Model 1 is more expensive than the mixture-averaged formulation. We also see that the possible benefit compared to the Dixon-Lewis formulation is to avoid the inversion of the matrix, which can be significant when $N$ is large. However, it implies the application of Eq. (8) or (9), where the latter does not bring any advantage since it implies a similar cost as the inversion of the $N \times N$ matrix (of the order of $N^{3}$ operations).

On the other hand, we can already anticipate the possible speed-up that can offer the $[1+M]^{(0)}$ model. First of all, the reduction of the number of operations compared to the standard Dixon-Lewis formulation is clear. Moreover, it is important to stress the fact that the binary diffusion coefficients are obtained through a third-order polynomial fit of their logarithm versus the logarithm of the temperature [7]:

$$
\ln \mathcal{D}_{i j}=\sum_{n=1}^{4} d_{n, i j}(\ln T)^{n-1}
$$

where $d_{n, i j}$ are the given fitting coefficients (satisfying $d_{n, i j}=$ $\left.d_{n, j i}\right)$. This implies that the evaluation of every binary diffusion coefficient includes the calculation of an exponential, which is an expensive operation in terms of CPU time. In this sense, the $[1+M]^{(0)}$ model can offer a significant speed-up compared to the mixture-averaged formulation, even if (depending on the value of $M$ ) the other parts of the evaluation of the fluxes require more operations.

\section{Resolution of one-dimensional steady and unsteady counterflow flamelets}

The 1D steady and unsteady solutions on the symmetry axis of the planar opposed-jet configuration problem are obtained by resolving the system of equations expressed in physical space, using the computer code LFLAM, developed at Ciemat (used for instance in [15]).

In physical space, we solve the continuity, momentum, species and temperature equations as described in $[16,17]$ for the planar geometry, here written in their unsteady form (in this formulation $F=\rho u$ and $G=-\rho v / y$ with $u$ the axial and $v$ the normal velocity components and $y$ the perpendicular direction):

$$
\begin{aligned}
& \frac{\partial \rho}{\partial t}=G-\frac{\partial F}{\partial x}, \\
& \frac{\partial G}{\partial t}=-F \frac{\partial}{\partial x}\left(\frac{G}{\rho}\right)+\frac{\partial}{\partial x}\left[\mu \frac{\partial}{\partial x}\left(\frac{G}{\rho}\right)\right]+H+\frac{G^{2}}{\rho}, \\
& \rho \overline{c_{p}} \frac{\partial T}{\partial t}=-\left[\overline{c_{p}} F+\sum c_{p k}\left(\rho Y_{k} V_{k}\right)\right] \frac{\partial T}{\partial x} \\
&+\frac{\partial}{\partial x}\left[\lambda \frac{\partial T}{\partial x}\right]-\sum h_{k} \dot{\omega}_{k}, \\
& \frac{\partial Y_{k}}{\partial t}=-F \frac{\partial Y_{k}}{\partial x}+\frac{\partial}{\partial x}\left[-\rho Y_{k} V_{k}\right]+\rho \dot{\omega}_{k},
\end{aligned}
$$

where the transport and thermodynamic coefficients, $\mu, \lambda, c_{p k}$, $h_{k}$ (resp. dynamic molecular viscosity, thermal conductivity, specific heat capacity of species $k$ and enthalpy of species $k$ ) are obtained from temperature-dependent polynomial fits in Chemkin format and where $\overline{c_{p}}$ is the specific heat capacity of the mixture. The mixture density $\rho$ is obtained from the ideal gas law at atmospheric pressure. The multicomponent diffusion fluxes $\rho Y_{k} V_{k}$ for species $k$ are obtained using either DixonLewis, mixture-averaged formulation, Model 1 or the $1+M$ model. The reaction rate of species $k$, last term in (23), is obtained from a given chemical mechanism in Chemkin format, as function of composition $Y_{k}$ and temperature $T$.

A fixed stagnation point is specified at the middle of the domain in order to discretise the continuity equation (20), and the boundary conditions are specified according to the potential flow assumption $(G=d F / d x=$ constant) on both sides (fuel "fu" and oxidiser "ox") of the $1 \mathrm{D}$ space, with: $G_{\mathrm{ox}}=-a . \rho_{\mathrm{ox}}$, $H=-G_{\mathrm{ox}}^{2} / \rho_{\mathrm{ox}}$ and $G_{\mathrm{fu}}=G_{\mathrm{ox}} \cdot\left(\rho_{\mathrm{fu}} / \rho_{\mathrm{ox}}\right)^{1 / 2}$. In this way, the strain rate $a$ is the parameter that defines the problem, together with the fuel and oxidiser composition and temperature conditions.

The system of equations (20)-(23) is solved either in its steady form, either in its unsteady form with a fixed time step $\Delta t$, using the algorithm "Twopnt program for boundary value problems" presented in [18]. By solving the above equations, we obtain the composition and temperature in physical space, $Y_{k}(x)$ and $T(x)$ (at discrete times in unsteady calculations). 
In the above system of equations, besides the multicomponent fluxes $\rho Y_{k} V_{k}$, it is interesting to note that the mixture viscosity $\mu$ is another possibly time-consuming property to be evaluated. The mixture viscosity is obtained here using the following semi-empirical formula (due to Wilke and modified by Bird et al) [7]:

$$
\begin{aligned}
& \mu=\sum_{k=1}^{N} \frac{X_{k} \mu_{k}}{\sum_{j=1}^{N} X_{j} \Phi_{k j}} \\
& \text { with } \Phi_{k j}=\left(1+\sqrt{\left.\frac{\mu_{k}}{\mu_{j}} \sqrt{\frac{W_{j}}{W_{k}}}\right)^{2} / \sqrt{8\left(1+\frac{W_{k}}{W_{j}}\right)},}\right.
\end{aligned}
$$

where $\mu_{k}$ are the single-component viscosities evaluated using polynomial fits of the form of (19). We see that the evaluation of the $N^{2}$ coefficients $\Phi_{k j}$ can be quite demanding. The definition of the $1+M$ main species could also be used to reduce the sums appearing in the above expression, reducing the number of coefficients $\Phi_{k j}$ to be evaluated to $(1+M)^{2}$ instead of $N^{2}$.

\section{Results}

\subsection{Steady counterflow flamelets}

We first consider steady flamelets in order to show the performance of the different multicomponent formulations.

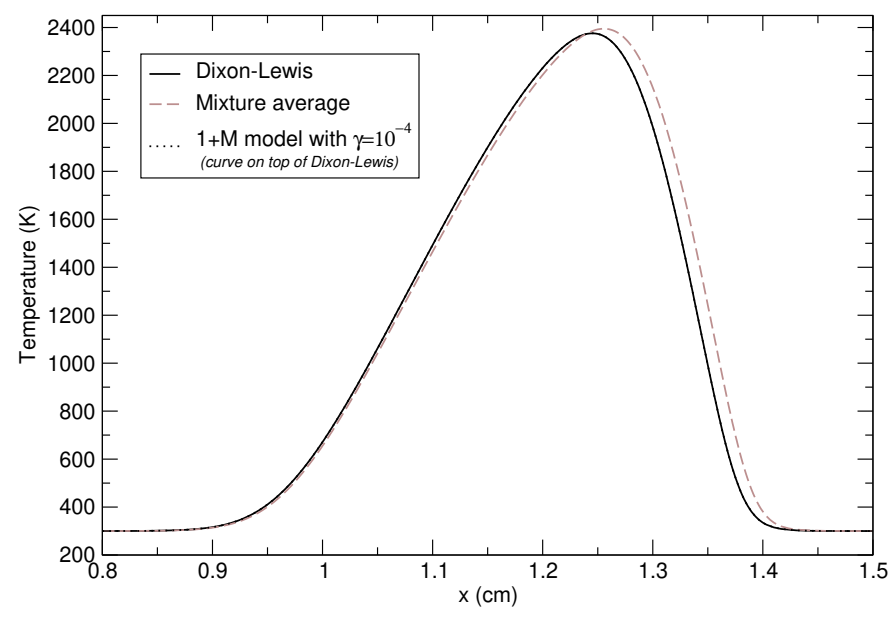

Figure 1: Steady counterflow flamelet of pure hydrogen in air at $300 \mathrm{~K}$ and strain rate $a=200 \mathrm{~s}^{-1}$ (with fuel boundary at $x=0$, oxidiser boundary at $x=2 \mathrm{~cm}$ and fixed stagnation point at $x=1 \mathrm{~cm}$ ). Temperature profile in physical space.

First, a simple case of pure hydrogen in air at $300 \mathrm{~K}$ is considered, at strain rate $a=200 \mathrm{~s}^{-1}$, using the chemical mechanism of [19] involving 9 species and 21 reactions. This is a case where differential diffusion effects are expected to be important, and therefore challenging for multicomponent diffusion models. Figure 1 shows that the mixture-averaged simplification is not exactly equivalent to Dixon-Lewis multicomponent diffusion. In this case, where the fuel is not diluted, we cannot obtain results with Model 1 with first-order or second-order truncation. As expected, a high order power series expansion would be needed in this case in order to obtain a reasonable approximation of multicomponent diffusion species fluxes, since the truncated power series expansion model was derived assuming the presence of a main diluting species. Results can be obtained in this case with the lowest order Model $1+M$ only at the condition that the threshold factor $\gamma$ in (10) is small, namely $\gamma<10^{-4}$. No solution is obtained when $\gamma \geq 10^{-3}$. This is due to the fact that with the threshold value $\gamma=10^{-3}$ the radical $\mathrm{H}$ is not included in the main species subset in some critical part of the flame, as will be discussed together with Figure 5. The obtained Model $1+M$ results corresponding to a $\gamma=10^{-4}$ threshold factor are in almost exact correspondence with Dixon-Lewis results.

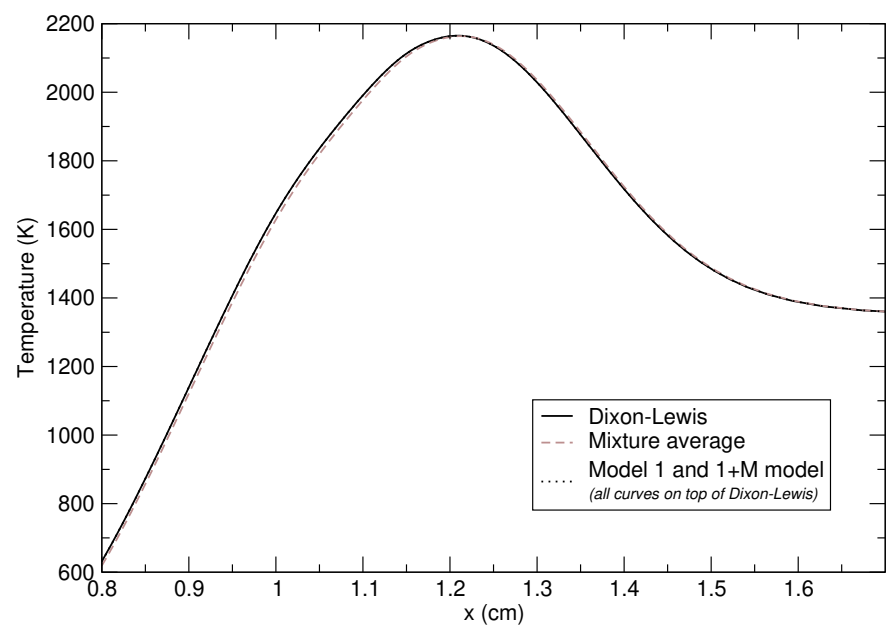

Figure 2: Steady diluted methane flamelet at strain rate $a=100 \mathrm{~s}^{-1}$ (with fuel boundary at $x=0$, oxidiser boundary at $x=2 \mathrm{~cm}$ and fixed stagnation point at $x=1 \mathrm{~cm})$. Temperature profile in physical space.

Second, we consider the steady solution corresponding to the conditions of the flame experimentally investigated by Cabra et al. [22]: a turbulent jet of methane/air mixture in a coflow of hot products of lean premixed hydrogen/air combustion, as summarised in Table 2. The chemical mechanism of [23] is

Table 2: Conditions of temperature and species mole fractions of fuel and hot coflow, corresponding to the atmospheric lifted $\mathrm{CH}_{4}$ turbulent jet flame of [22].

\begin{tabular}{|l|l|l|}
\hline & Fuel & Hot coflow \\
\hline Temperature & $T=320 K$ & $T=1350 K$ \\
\hline Mole fractions & $X_{\mathrm{CH}_{4}}=0.33$ & $X_{\mathrm{H}_{2} \mathrm{O}}=0.15$ \\
& $X_{\mathrm{O}_{2}}=0.15$ & $X_{\mathrm{O}_{2}}=0.12$ \\
& $X_{\mathrm{N}_{2}}=0.52$ & $X_{\mathrm{N}_{2}}=0.73$ \\
\hline
\end{tabular}

used, involving 57 species and 268 reactions. As can be seen in Table 2, we now consider a case where $\mathrm{N}_{2}$ is a main diluting non-reacting species everywhere in the flame. This is a very favourable case for the truncated power series model. In this case, results are now obtained with Model 1, or with the lowest order Model $1+M$ even with $\gamma=10^{-2}$, and the results are identical to the results obtained with the exact Dixon-Lewis formulation. Figure 2 illustrates the very small differences be- 
tween mixture-averaged and Dixon-Lewis results for the steady solution.

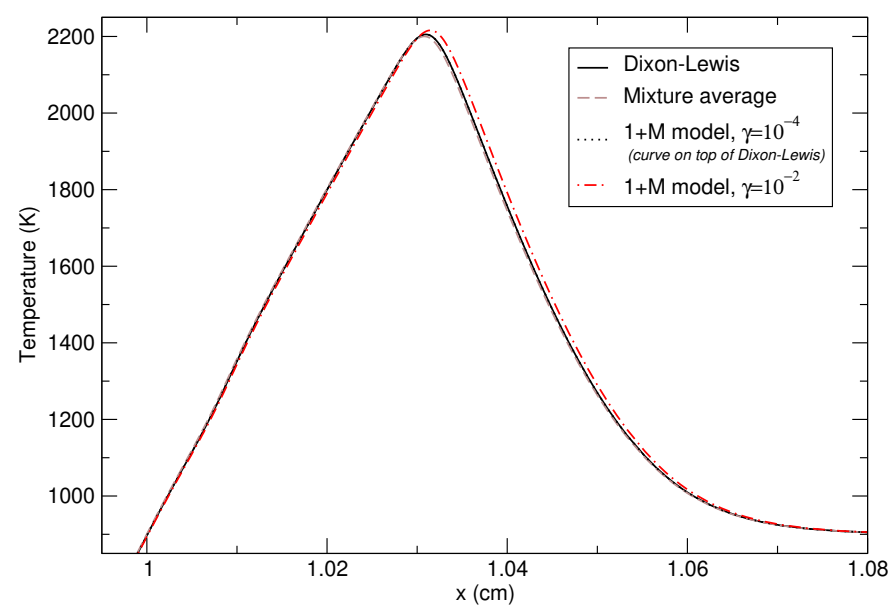

Figure 3: Steady counterflow flamelet of dodecane at high pressure and strain rate $a=100 \mathrm{~s}^{-1}$ (with fuel boundary at $x=0$, oxidiser boundary at $x=2 \mathrm{~cm}$ and fixed stagnation point at $x=1 \mathrm{~cm}$ ). Temperature profile in physical space.

A very different case is considered: pure $n$-dodecane at $363 \mathrm{~K}$, in a mixture of oxigen/nitrogen $(0.15: 0.85$ by volume $)$ at $900 \mathrm{~K}$, at a pressure of $5.06 \mathrm{MPa}$. This corresponds to the experimental conditions of the so-called "Spray A" when $n$-dodecane is used as a diesel surrogate fuel, considered as a reference case in the Engine Combustion Network (ECN) [20]. In this case, the chemical mechanism of [21] is used, involving 255 species and 1509 reactions. Figure 3 shows that the mixtureaveraged results are very close to Dixon-Lewis (although not exactly identical). This is again a case where the fuel is not diluted and where no results can be obtained with the first-order or second-order truncated power series expansions (Model 1). However, results can be obtained with the lowest order Model $1+M$ with $\gamma=10^{-2}$, leading to some small differences. With lower values of $\gamma$ results get closer to Dixon-Lewis, and are identical when $\gamma=10^{-4}$.

These first cases already show the limitations of the truncated power series expansion models in practical calculations, when no main diluting species can be defined. In general, high order truncations may be needed, and the evaluation of $c_{i j}^{(p)}$ (truncated at order $p>1$ ) will be more expensive than directly inverting the matrix in (4). As expected, the mixture-averaged simplification differs from Dixon-Lewis when differential diffusion effects are important. On the other hand, the lowest order Model $1+M$ appears to be a very good approximation of Dixon-Lewis multicomponent diffusion, when the threshold factor $\gamma$ is small enough.

\subsection{Diluted methane counterflow igniting flamelet}

Finally, we consider an unsteady igniting methane flamelet starting from the inert solution corresponding to the conditions summarised in Table 2. This is a transient problem where different components play a role in the ignition process, like for instance $\mathrm{HO}_{2}, \mathrm{H}_{2} \mathrm{O}_{2}, \mathrm{CH}_{2} \mathrm{O}$ or $\mathrm{C}_{2} \mathrm{H}_{6}$, but also $\mathrm{C}_{2} \mathrm{H}_{2}$ or $\mathrm{C}_{2} \mathrm{H}_{4}$.

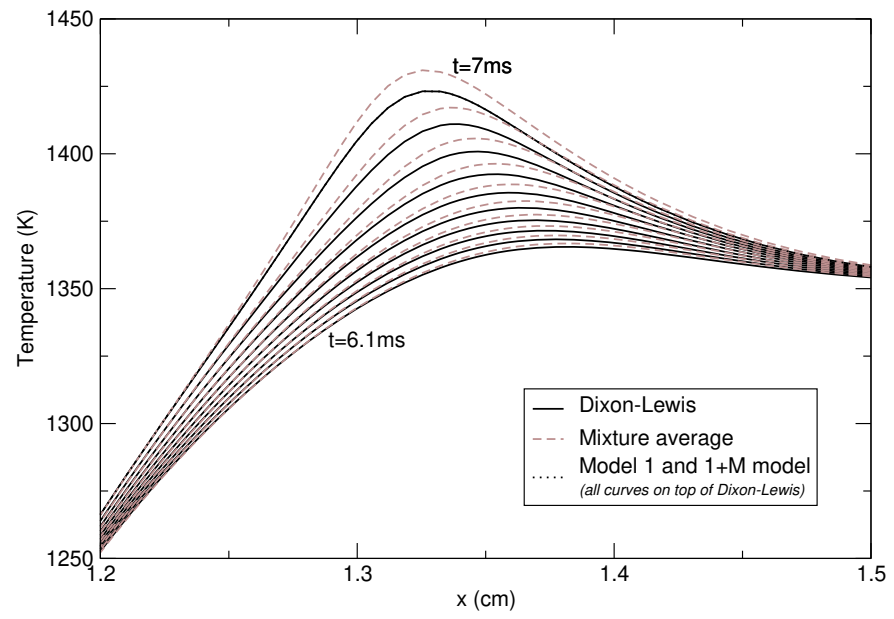

Figure 4: Igniting diluted methane flamelet at strain rate $a=100 \mathrm{~s}^{-1}$ (with fuel boundary at $x=0$, oxidiser boundary at $x=2 \mathrm{~cm}$ and fixed stagnation point at $x=1 \mathrm{~cm}$ ). Temperature profile in physical space at discrete times (every $10^{-4} \mathrm{~s}$ between $6.1 \mathrm{~ms}$ and $7 \mathrm{~ms}$ ).

This is therefore a more challenging case compared to the steady results shown in Figure 2. For the different approximations of Model 1, and Model $[1+M]^{(0)}$, we can verify in Figure 4 the very good correspondence with the exact Dixon-Lewis formulation already observed in the steady case. However, the limitation of the mixture-averaged approximation now appears more clearly: in this unsteady problem, the different radicals appearing during the ignition process imply differential diffusion effects which are not represented accurately by this approximation.

\subsection{Relative computational cost of the multicomponent diffu- sion approaches}

In Table 3, for the three steady cases considered, we summarise the relative cost of the evaluation of multicomponent fluxes for all multicomponent diffusion approaches: DixonLewis "D.-L.", mixture-averaged "Mix.-av.", $[1+M]^{(0)}$ with different values for $\gamma$, and when available, Model 1 with 1st order and 2 nd order corrections. The computational cost is normalised by the time required for the evaluation of the mixture viscosity $\mu$ using (24). For this comparison, in the $[1+M]^{(0)}$ model calculations where the evaluation of $\mu$ is improved by only considering the $1+M$ main species, we solve (24) as an extra equation considering the $N$ species in order to normalise the computational times.

Considering Model 1, in the steady diluted methane flame where results can be obtained with truncated power series, we see that the first order truncation is less efficient than the mixture-averaged formulation, as expected from Table 1. Moreover, the second-order truncation appears to have a similar cost as Dixon-Lewis, showing that in this case the evaluation of the approximated dimensionless coefficients $c_{i j}^{(2)}$ in (9) (expected to be of the same order $O\left(N^{3}\right)$ in Table 1) is as expensive as directly inverting the matrix $(\mathbb{1}+A)_{i j}$ in (4).

On the other hand, the computational cost of Model $[1+M]^{(0)}$ is lower than the mixture-averaged formulation when the num- 
Table 3: Relative computational cost for the evaluation of the multicomponent species fluxes normalised by the time required for the evaluation of the mixture viscosity $\mu$ using (24), for the three steady cases considered: pure $\mathrm{H}_{2}$ flame (9 species), diluted $\mathrm{CH}_{4}$ flame (57 species) and pure dodecane flame ( 255 species). The additional relative time for evaluation of $\mu$ is indicated (taking advantage of the definition of the $1+M$ main species when possible). The details on the time spent on each step corresponding to the three lines appearing in Table 1 are first specified. The mean number of main species $\langle 1+M\rangle$ and maximum number of main species $\max (1+M)$ in $[1+M]^{(0)}$ model calculations with $\gamma=10^{-4}$ and $\gamma=10^{-5}$ are indicated, and can be compared to the total number of species $N$.

\begin{tabular}{|c|c|c|c|c|c|c|}
\hline & D.-L. & Mix.-av. & $\gamma=10^{-4}$ & $\gamma=10^{-5}$ & Model 1 (1st order) & (2nd order) \\
\hline$N=9$ & & & $\begin{array}{c}\langle 1+M\rangle=4.4 \\
\max (1+M)=7\end{array}$ & $\begin{array}{c}\langle 1+M\rangle=5.2 \\
\max (1+M)=9\end{array}$ & & \\
\hline $\mathcal{D}_{i j}$ & 0.32 & 0.32 & 0.29 & 0.31 & & \\
\hline$c_{i j}\left(\right.$ or $\left.D_{i}\right)$ & 0.81 & 0.15 & 0.33 & 0.40 & & \\
\hline fluxes & 0.076 & 0.045 & 0.064 & 0.066 & & \\
\hline $\begin{array}{c}\rho Y_{i} \mathbf{V}_{i} \\
\mu\end{array}$ & $\begin{array}{c}1.21 \\
+1.0\end{array}$ & $\begin{array}{c}0.51 \\
+1.0\end{array}$ & $\begin{array}{c}0.68 \\
+0.360\end{array}$ & $\begin{array}{c}0.78 \\
+0.472\end{array}$ & & \\
\hline Total & $100 \%$ & $68 \%$ & $47 \%$ & $57 \%$ & & \\
\hline$N=57$ & & & $\begin{array}{c}\langle 1+M\rangle=11.2 \\
\max (1+M)=16\end{array}$ & $\begin{array}{c}\langle 1+M\rangle=15.0 \\
\max (1+M)=22\end{array}$ & & \\
\hline $\mathcal{D}_{i j}$ & 0.34 & 0.34 & 0.16 & 0.20 & 0.34 & 0.34 \\
\hline$c_{i j}\left(\right.$ or $\left.D_{i}\right)$ & 1.74 & 0.15 & 0.20 & 0.29 & 0.28 & 1.60 \\
\hline fluxes & 0.097 & 0.0066 & 0.038 & 0.048 & 0.097 & 0.097 \\
\hline $\begin{array}{c}\rho Y_{i} \mathbf{V}_{i} \\
\mu\end{array}$ & $\begin{array}{l}2.17 \\
+1.0\end{array}$ & $\begin{array}{r}0.50 \\
+1.0\end{array}$ & $\begin{array}{c}0.39 \\
+0.056\end{array}$ & $\begin{array}{c}0.54 \\
+0.095\end{array}$ & $\begin{array}{c}0.72 \\
+1.0\end{array}$ & $\begin{array}{r}2.04 \\
+1.0\end{array}$ \\
\hline Total & $100 \%$ & $47 \%$ & $14 \%$ & $20 \%$ & $54 \%$ & $96 \%$ \\
\hline$N=255$ & & & $\begin{array}{c}\langle 1+M\rangle=23.3 \\
\max (1+M)=49\end{array}$ & $\begin{array}{c}\langle 1+M\rangle=32.0 \\
\max (1+M)=63\end{array}$ & & \\
\hline $\mathcal{D}_{i j}$ & 0.35 & 0.35 & 0.08 & 0.11 & & \\
\hline$c_{i j}\left(\right.$ or $\left.D_{i}\right)$ & 6.58 & 0.15 & 0.15 & 0.23 & & \\
\hline fluxes & 0.176 & 0.0016 & 0.024 & 0.031 & & \\
\hline $\begin{array}{c}\rho Y_{i} \mathbf{V}_{i} \\
\mu\end{array}$ & $\begin{array}{r}7.12 \\
+1.0\end{array}$ & $\begin{array}{r}0.51 \\
+1.0\end{array}$ & $\begin{array}{c}0.26 \\
+0.017\end{array}$ & $\begin{array}{c}0.37 \\
+0.029\end{array}$ & & \\
\hline Total & $100 \%$ & $19 \%$ & $3.4 \%$ & $4.9 \%$ & & \\
\hline
\end{tabular}

ber of species considered is large. This is quite remarkable, and we can clearly see that the main gain comes from the evaluation of fewer binary coefficients $\mathcal{D}_{i j}$. This is of course related to the number $1+M$ of main species considered in each case (here with rather small values for the threshold factor $\gamma$ ). The mean value $\langle 1+M\rangle$ and maximum value of the number of main species are specified in the table and can be compared to the number $N$ of species considered. This will be further detailed in the next section.

Model $[1+M]^{(0)}$ also appears to offer an extra advantage for the evaluation of the mixture viscosity $\mu$ when only considering the $1+M$ main species in Eq. (24), instead of the total number $N$ of species. It takes less than half the time for a small number of species like in the hydrogen case, but the cost can be reduced by a factor 25 in the methane case, and by 30 or 50 in the dodecane case. This extra advantage makes the $[1+M]^{(0)}$ model always more efficient than the mixture-averaged formulation when considering the total time required to evaluate both $\rho Y_{i} \mathbf{V}_{i}$ and $\mu$, even when the number of species considered is rather small.

The times appearing in Table 3 concerning the final evaluation of the diffusion fluxes according to Eq. (1), (3), (15) or (17) (line "fluxes") are small compared to the others. However, as explained in Appendix A, when a Jacobian matrix needs to be evaluated numerically, this final evaluation of the diffusion fluxes needs to be performed many times (by considering perturbations of the current solution vector). This is the case for the "Twopnt" algorithm used to solve the problem. In Appendix A, we detail the efficient implementation used in the code LFLAM in order to avoid a too time consuming evaluation of the Jacobian matrix when $N$ is large.

\subsection{Main species in physical space}

In order to better illustrate why the Model $1+M$ can be so efficient, it is useful to look at the distribution of the main species in physical space for the different cases considered. Figures 5, 6 and 7 show the distribution in physical space of the number $1+M$ of main species for the three steady flamelets considered. The temperature profiles already plotted in Figures 1, 2 and 3 are also reproduced together with the grid nodes.

We can clearly see how in the fuel and oxidiser streams the number of main species reduces to the number of species in the streams ( 1 in pure fuel, 2 in air and 3 in the case of diluted methane in hot coflow). Moreover, we can observe how the number of main species increases in the flame zone. Note that 


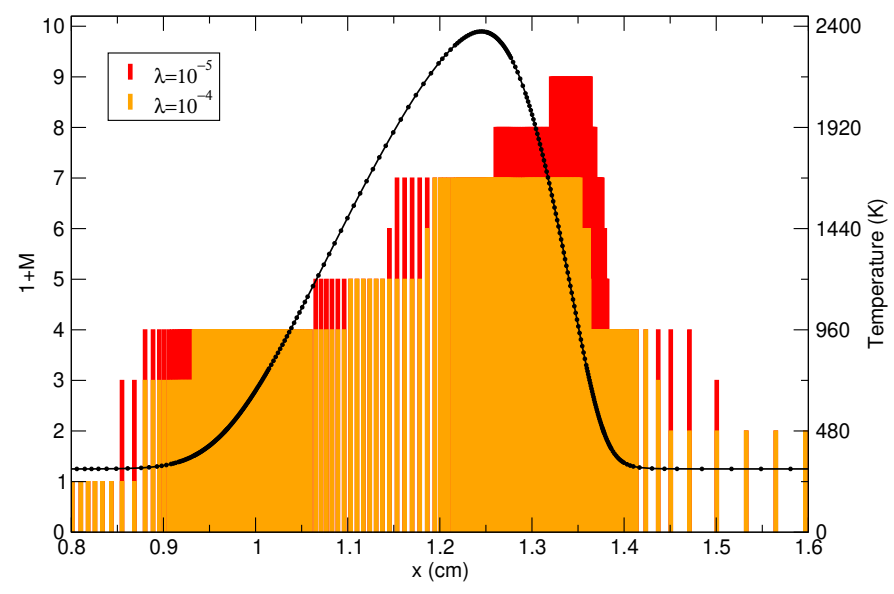

Figure 5: Number of main species at cell centers for different values of $\lambda$ in Model $[1+M]^{(0)}$, for the evaluation of $\rho Y_{i} \mathbf{V}_{i}$ (and $\mu$ ) in the pure hydrogen steady flamelet corresponding to Figure 1.

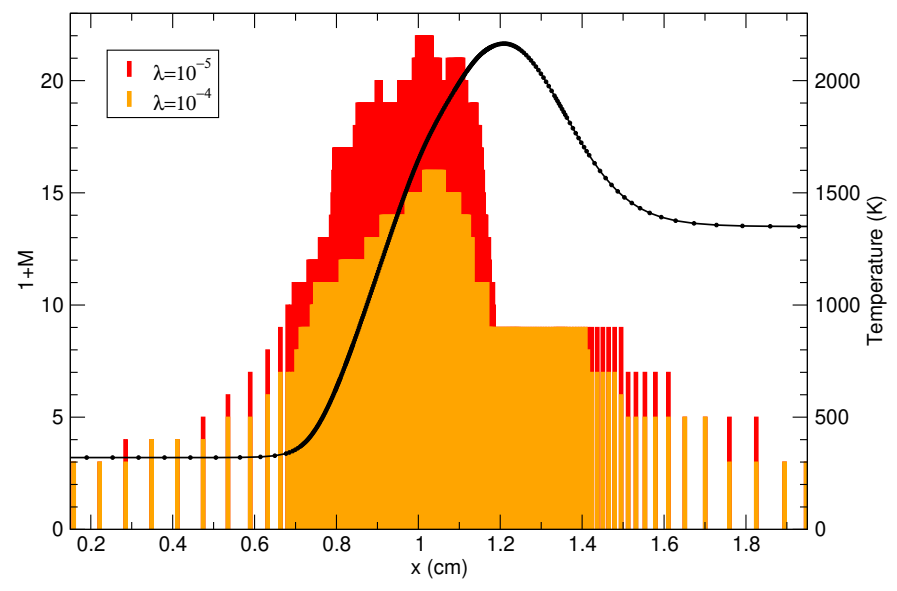

Figure 6: Number of main species at cell centers for different values of $\lambda$ in Model $[1+M]^{(0)}$, for the evaluation of $\rho Y_{i} \mathbf{V}_{i}$ (and $\mu$ ) in the diluted methane steady flamelet corresponding to Figure 2 .

in the pure hydrogen case, it can locally reach the total number of species $N=9$ on the lean side when $\gamma=10^{-5}$.

In the pure hydrogen case, we can also understand the reason why no results are obtain when $\gamma \geq 10^{-3}$. We verified that a solution is obtained when the threshold factor $\gamma$ is set to $10^{-3}$ for all species, except for the radical $\mathrm{H}$ for which $\gamma$ is set to $10^{-4}$. This tells us that with the threshold value $\gamma=10^{-3}$, the radical $\mathrm{H}$ is removed from the main species subset in some part of the flame where it is necessary to include this species. This is in line with the observation of Xin et al. [14] where they verify that their heptane-air combustion system is sensitive to the diffusivity of a subset of critical-diffusivity-species which are "either in high concentration or are higly reactive radicals such as $\mathrm{H}$ and $\mathrm{OH}$ ".

This suggests a refinement of criterion (10) used to identify the main species:

$$
X_{i} \geq \gamma_{i} \cdot X_{K}
$$

where the threshold factor $\gamma_{i}$ can be defined with a different value for each species $i$. With such a species-dependent crite-

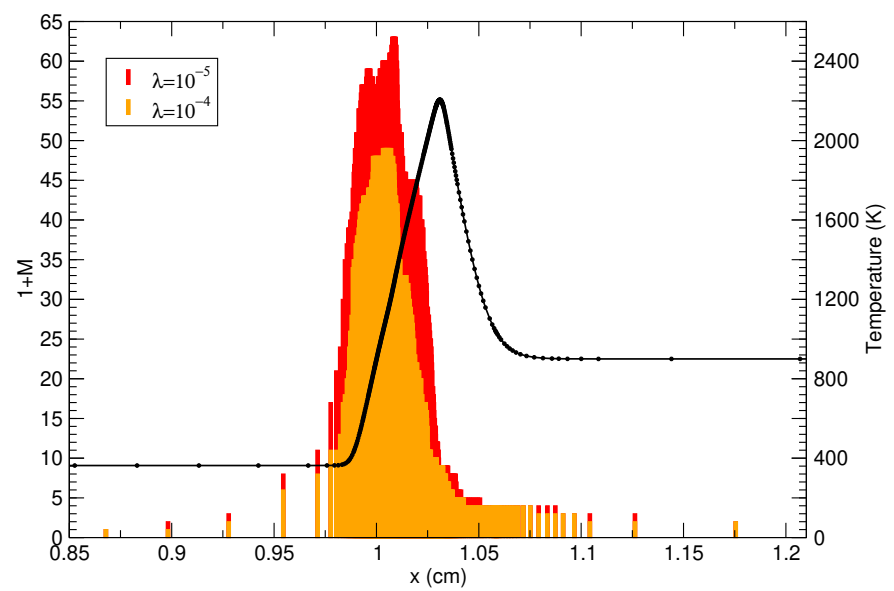

Figure 7: Number of main species at cell centers for different values of $\lambda$ in Model $[1+M]^{(0)}$, for the evaluation of $\rho Y_{i} \mathbf{V}_{i}$ (and $\mu$ ) in the pure dodecane steady flamelet corresponding to Figure 3 .

rion, it would be possible to further improve the efficiency of the proposed $[1+M]^{(0)}$ model, by possibly using large threshold values for most species and smaller threshold values for the higly reactive radicals. In the present formulation, we can claim that it is safe to use a global threshold value of $\gamma=10^{-5}$.

Figures 1, 2 and 3 also allow to picture the benefits that the Model $[1+M]^{(0)}$ will offer in realistic configurations of $3 \mathrm{D}$ flames: it will drastically reduce the number of main species in the unmixed inlet streams in diffusion flames, or in the fresh gases and burnt gases in premixed flames, and the maximum number of main species will be localised in the flame zone. This will allow to evaluate the Dixon-Lewis multicomponent diffusion fluxes $\rho Y_{i} \mathbf{V}_{i}$ at a similar or lower cost than when using the mixture-averaged formulation. Moreover, it will also be possible to evaluate the mixture viscosity $\mu$ in a much more efficient way.

On the other hand, since the gain in computational time is mainly localised out of the flame zone, strategies will need to be designed in the case of parallel computations based on domain decomposition in order to get a good load balance.

\section{Conclusions}

Different multicomponent diffusion formulations have been compared, neglecting the effect of thermal diffusion (Soret effect). The comparisons are made in simple one-dimensional steady and unsteady configurations (counterflow diffusion flames), which include relevant physical issues that may appear in Direct Numerical Simulations of practical flames.

The known limitations of the mixture-averaged simplification are illustrated in a pure hydrogen flame, where differential diffusion effects are important, but also in a diluted methane igniting flamelet where the different radicals appearing during the ignition process need to be correctly modelled.

It appears that the truncated power series expansion model first proposed by Arias-Zugasti et al. [1] (Model 1), would require high order truncations in order to be valid when no main 
diluting species can be defined (for instance when pure fuel diffusion flames are considered). Moreover, in the diluted methane case (involving 57 species), the cost of the second order truncation appears to be similar to the cost of the implementation of Dixon-Lewis formulation with direct inversion of the $N \times N$ matrix.

The lowest order Model $1+M$, which takes advantage of the lowest order truncation formulation only for the most diluted species, appears to be very efficient (even more efficient than mixture-averaged diffusion when the number of species is large), and leads to identical results as a direct implementation of Dixon-Lewis diffusion when the threshold factor $\gamma$ is small enough. This is mainly due to a reduced number of binary coefficients $\mathcal{D}_{i j}$ to be evaluated. Moreover, we show that the definition of $1+M$ main species can also greatly reduce the cost of the time-consuming evaluation of the mixture viscosty $\mu$. Although the purpose of the present paper is to derive an efficient multicomponent diffusion model corresponding to the Dixon-Lewis formulation, we describe in Appendix B how the definition of the $1+M$ main species can also be used in order to derive a reduced mixture-averaged formulation.

In the future, the proposed formulation could be further improved by introducing a species-dependent threshold factor $\gamma_{i}$ as in (25) in order to define the subset of main species, providing a simple criterion in order to define the critical-diffusivityspecies (CDS) proposed by Xin et al. [14]. In the present form of the model, we propose the value $\gamma=10^{-5}$ as a safe global threshold value to be used.

The distribution of the number of main species in the modelled flames shows that the cost reduction is the most important away from the flame zone. This indicates the potential of the Model $[1+M]^{(0)}$ for the simulation of realistic 3D flames. This also indicates that in the case of parallel computations, special attention will need to be paid to domain decomposition in order to take full advantage of the proposed method.

\section{Acknowledgments}

This work is supported at Ciemat by the project \#ENE201565852-C2-2-R (MINECO/ FEDER, EU), and supported at UNED (Madrid) by Ministerio de Ciencia e Innovación (\#CSD2010-00011 and \#ENE2011-26868), Comunidad de Madrid (\#S2009/ENE-1597) and Ministerio de Economía y Competitividad (\#ENE2015-67635-R).

\section{A. Note on the numerical evaluation of the Jacobian matrix}

The algorithm "Twopnt program for boundary value problems" [18] used in order to obtain the results presented in this paper requires the numerical evaluation of the Jacobian matrix of the problem to be solved described by the system of equations (20)-(23). This system of $N+3$ equations can be written in the form:

$$
\mathcal{F}_{i}(\boldsymbol{\Phi})=0, \quad i=1, N+3
$$

with $\boldsymbol{\Phi}=(F, G, T, \mathbf{Y})$. The Jacobian matrix that needs to be evaluated in order to solve the problem written in the compact form (26) is $\left[\partial \mathcal{F}_{i} / \partial \Phi_{j}\right]$. The $(N+3)^{2}$ derivatives in the Jacobian matrix are evaluated numerically as follows:

$$
\begin{aligned}
\frac{\partial \mathcal{F}_{i}}{\partial \Phi_{j}} & =\frac{\mathcal{F}_{i}\left(\ldots, \Phi_{j}+\epsilon_{j}, \ldots\right)-\mathcal{F}_{i}\left(\ldots, \Phi_{j}, \ldots\right)}{\epsilon_{j}} \\
\text { with } & \epsilon_{j}=\Phi_{j} . \epsilon_{r}+\epsilon_{a}
\end{aligned}
$$

where $\epsilon_{r}$ and $\epsilon_{a}$ are the relative and absolute perturbations, respectively.

Table 4: Relative time (normalised as in Table 3) dedicated to the final evaluation of the diffusion fluxes in the evaluation of the Jacobian matrix, both in the efficient implementation considered here and in a unefficient straightforward implementation.

\begin{tabular}{|ll|c|c|c|}
\hline & & $N=9$ & $N=57$ & $N=255$ \\
\hline D.-L. & efficient & 0.50 & 0.45 & 0.586 \\
& straightforward & 2.77 & 17.37 & 124.14 \\
\hline Mix.-av. & efficient & 0.23 & 0.082 & 0.074 \\
& straightforward & 1.60 & 1.15 & 1.12 \\
\hline$\gamma=10^{-4}$ & efficient & 0.41 & 0.23 & 0.252 \\
& straightforward & 2.33 & 6.69 & 16.85 \\
\hline$\gamma=10^{-5}$ & efficient & 0.42 & 0.26 & 0.276 \\
& straightforward & 2.40 & 8.02 & 21.89 \\
\hline
\end{tabular}

In the evaluation of the Jacobian matrix, all the mixture and pure species properties and coefficients, $\mu, \lambda, \overline{c_{p}}, c_{p k}, h_{k}, \mathcal{D}_{i j}$, $c_{i j}$ (or $D_{i}$ ) are kept unperturbed in the $N+3$ equations $\mathcal{F}_{i}(\boldsymbol{\Phi})$. In this way, there is no need to evaluate the "perturbed" binary coefficients $\mathcal{D}_{i j}$ and dimensionless coefficients $c_{i j}$ (first two lines in Table 1). However the perturbed diffusion fluxes $\rho Y_{k} \mathbf{V}_{k}$ need to be evaluated according to Eq. (1), (3), (15) or (17) including the perturbations in $Y_{k}$.

At each grid point of the one-dimensional domain, in an staightforward implementation of the Jacobian matrix the perturbed diffusion fluxes would be evaluated $n_{x}(N+3)$ times, by repeating the complete evaluation of the perturbed diffusion fluxes after perturbing each of the $N+3$ component of $\boldsymbol{\Phi}$, and considering the pattern of $n_{x}$ points for the finite-difference discretisation of the derivatives in the equations $\mathcal{F}_{i}(\boldsymbol{\Phi})$ (here $\left.n_{x}=3\right)$.

A more careful and more efficient implementation consists in only evaluating the $N$ perturbed diffusion fluxes, by simply adding to the unperturbed fluxes the modification implied by the perturbation $\epsilon_{k}$ of every mass fraction $Y_{k}$, with no need to consider the whole sums given by Eq. (1), (3), (15) or (17). For instance, instead of considering Eq. (3), the perturbed fluxes $\left[\rho Y_{k} \mathbf{V}_{k}\right]^{*, j}$ corresponding to the perturbation $Y_{j}+\epsilon_{j}$ are simply obtained from the unperturbed fluxes $\rho Y_{k} \mathbf{V}_{k}$ as follows:

$$
\left[\rho Y_{k} \mathbf{V}_{k}\right]^{*, j}=\rho Y_{k} \mathbf{V}_{k}-\rho \frac{W_{k}}{\bar{W}} \mathcal{D}_{k N} c_{k j} \nabla\left(\left[X_{j}\right]^{*}-X_{j}\right)
$$

where $\left[X_{j}\right]^{*}-X_{j}$ is the pertubation of the mole fraction corresponding to the perturbation $\epsilon_{j}$ of the mass fraction $Y_{j}$. The 
same can be written for Eq. (1), (15) or (17). This becomes of course quite relevant when $N$ is large.

In Table 4, we compare the relative times dedicated to the perturbed diffusion fluxes in the evaluation of the Jacobian matrix (times normalised by the time required for the evaluation of $\mu$ as in Table 3$)$. We verify that the "straightforward" time is indeed about $n_{x}(N+3)$ times the reference time reported in Table 3 . We can observe the importance of a careful implementation, in particular when $N$ is large.

\section{B. Reduced mixture-averaged model}

Table 5: Relative computational cost for the evaluation of the multicomponent species fluxes (see caption in Table 3): standard mixture-averaged model "Mix.av.", proposed $[1+M]^{(0)}$ model with $\gamma=10^{-5}$ and reduced mixture-averaged model "New Mix.-av." with $\gamma=10^{-5}$.

\begin{tabular}{|c|c|c|c|}
\hline & Mix.-av. & {$[1+M]^{(0)}$} & New Mix.-av. \\
\hline$N=9$ & & $\begin{array}{c}\langle 1+M\rangle=5.2 \\
\rightarrow \max =9\end{array}$ & $\begin{array}{c}\langle 1+M\rangle=5.3 \\
\rightarrow \max =9\end{array}$ \\
\hline $\mathcal{D}_{i j}$ & 0.32 & 0.31 & 0.31 \\
\hline$c_{i j}\left(\right.$ or $\left.D_{i}\right)$ & 0.15 & 0.40 & 0.09 \\
\hline fluxes & 0.045 & 0.066 & 0.045 \\
\hline $\begin{array}{c}\rho Y_{i} \mathbf{V}_{i} \\
\mu\end{array}$ & $\begin{array}{r}0.51 \\
+1.0\end{array}$ & $\begin{array}{c}0.78 \\
+0.472\end{array}$ & $\begin{array}{c}0.45 \\
+0.499\end{array}$ \\
\hline Total & $100 \%$ & $83 \%$ & $63 \%$ \\
\hline$N=57$ & & $\begin{array}{c}\langle 1+M\rangle=15.0 \\
\rightarrow \max =22\end{array}$ & $\begin{array}{c}\langle 1+M\rangle=15.0 \\
\rightarrow \max =22\end{array}$ \\
\hline $\mathcal{D}_{i j}$ & 0.34 & 0.20 & 0.20 \\
\hline$c_{i j}\left(\right.$ or $\left.D_{i}\right)$ & 0.15 & 0.29 & 0.043 \\
\hline fluxes & 0.0066 & 0.048 & 0.0067 \\
\hline $\begin{array}{c}\rho Y_{i} \mathbf{V}_{i} \\
\mu\end{array}$ & $\begin{array}{r}0.50 \\
+1.0\end{array}$ & $\begin{array}{c}0.54 \\
+0.095\end{array}$ & $\begin{array}{c}0.25 \\
+0.095\end{array}$ \\
\hline Total & $100 \%$ & $42 \%$ & $23 \%$ \\
\hline$N=255$ & & $\begin{array}{c}\langle 1+M\rangle=32.0 \\
\rightarrow \max =63\end{array}$ & $\begin{array}{c}\langle 1+M\rangle=31.9 \\
\rightarrow \max =63\end{array}$ \\
\hline $\mathcal{D}_{i j}$ & 0.35 & 0.11 & 0.11 \\
\hline$c_{i j}\left(\right.$ or $\left.D_{i}\right)$ & 0.15 & 0.23 & 0.020 \\
\hline fluxes & 0.0016 & 0.031 & 0.0016 \\
\hline $\begin{array}{c}\rho Y_{i} \mathbf{V}_{i} \\
\mu\end{array}$ & $\begin{array}{r}0.51 \\
+1.0\end{array}$ & $\begin{array}{c}0.37 \\
+0.029\end{array}$ & $\begin{array}{c}0.13 \\
+0.029\end{array}$ \\
\hline Total & $100 \%$ & $26 \%$ & $11 \%$ \\
\hline
\end{tabular}

Table 5, compares the relative costs of the standard mixture averaged model and $[1+M]^{(0)}$ model with $\gamma=10^{-5}$, already reported in Table 3, to the cost of a reduced mixture averaged model that would take full advantage of the definition of $1+M$ main species with $\gamma=10^{-5}$ (reduced number of binary diffusion coefficients $\mathcal{D}_{i j}$ to be evaluated, reduced sum in (16), and reduced sums in the evaluation of $\mu$ in (24)).

We can see how the definition of the $1+M$ main species indeed allows to define a more efficient reduced mixture-averaged model, in particular when $N$ is large.

\section{References}

[1] Arias-Zugasti, M., Garcia-Ybarra, P.L., Castillo, J.L., Efficient calculation of multicomponent diffusion fluxes based on kinetic theory, Combust. Flame 163:540-556 (2016).

[2] Chapman, S., Cowling, T.G., The Mathematical Theory of Non-Uniform Gases, Cambridge University Press, Cambridge UK, 1985.

[3] Hirschfelder, J.O., Curtiss, C.F., Bird, R.B., The Molecular Theory of Gases and Liquids, Wiley, New York NY, 1964.

[4] Ferziger, J.H., Kaper, H.G., Mathematical Theory of Transport Processes in Gases, North-Holland Publishing Company, Amsterdam, 1972.

[5] Dixon-Lewis, G., Flame structure and flame reaction kinetics II. Transport phenomena in multicomponent systems, Proc. Roy. Soc. A. 304:111-135 (1968).

[6] Monchick, L., Mason, E.A., Transport Properties of Polar Gases, J. Chem. Phys. 35:1676-1697 (1961).

[7] Kee, R.J., Rupley, F.M., Miller, J.A., Coltrin, M.E., Grcar, J.F., Meeks, E., Moffat, H.K., Lutz, A.E., Dixon-Lewis, G., Smooke, M.D., Warnatz, J., Evans, G.H., Larson, R.S., Mitchell, R.E., Petzold, L.R., Reynolds, W.C., Caracotsios, M., Stewart, W.E., Glarborg, P., Wang, C., Adigun, O., CHEMKIN Collection, Reaction Design, Inc., San Diego, CA (2000).

[8] Hirschfelder, J.O., Curtiss, C.F., Bird, R.B., The molecular theory of gases and liquids, Wiley, 1954.

[9] Garcia-Ybarra, P., Nicoli, C., Clavin, P., Soret and dilution effects on premixed flames, Combust. Sci. Technol. 42:87-109 (1984).

[10] Jones, W.W., Boris, J.P., An algorithm for multispecies diffusion fluxes, Computers and Chemistry 5:139-146 (1981).

[11] Oran, E.S., Boris, J.P., Detailed modeling of combustion systems, Prog. Energy Combust. Sci. 7:1-72 (1981).

[12] Giovangigli, V., Convergent iterative Methods for Multicomponent Diffusion, Impact Comput. Sci. Eng. 3:244-276 (1991).

[13] Ern, A., Giovangigli, V., Multicomponent transport algorithms, Lecture Notes in Physcis Monographs, Springer, Berlin, Heidelberg (2008).

[14] Xin, Y., Liang, W., Liu, W., Lu, T., Law, C.K., A reduced multicomponent diffusion model, Combust. Flame 162:68-74 (2015).

[15] Naud, B., Novella, R., Pastor, J.-M., Winklinger, J.F., RANS modelling of a lifted $\mathrm{H}_{2} / \mathrm{N}_{2}$ flame using an unsteady flamelet progress variable approach with presumed PDF Combust. Flame 162:893-906 (2015).

[16] Kee, R.J., Miller, J.A., Evans, G.H., Dixon-Lewis, G., A computational model of the structure and extinction of strained, opposed flow, premixed methane-air flames Proc. Comb. Inst. 22:1479-1494 (1989).

[17] Lutz, A.E., Kee, R.J., Grcar, J.F., Rupley, F.M., OPPDIF: A Fortran program for computing opposed-flow diffusion flames, Sandia National Laboratories Report, SAND96-8243 (1997).

[18] Grcar, J.F., The Twopnt Program for Boundary Value Problems, Sandia National Laboratories Report, SAND91-8230 (1992).

[19] Saxena, P., Williams, F.A., Testing a small detailed chemical-kinetic mechanism for the combustion of hydrogen and carbon monoxide, Combust. Flame 145:316-323 (2006).

[20] Pickett L. M., Bruneaux G., Payri R., Engine combustion network. Sandia National Laboratories, Livermore, CA, https://ecn.sandia.gov/ (2018).

[21] Narayanaswamy, K., Pepiot, P., Pitsch, H., A chemical mechanism for low to high temperature oxidation of $n$-dodecane as a component of transportation fuel surrogates, Combust. Flame 161:866-884 (2014).

[22] Cabra, R., Chen, J.-Y., Dibble, R.W., Karpetis, A.N., Barlow, R.S., Lifted methane-air jet flames in a vitiated coflow, Combust. Flame 143:491-506 (2005).

[23] "Chemical-Kinetic Mechanisms for Combustion Applications", San Diego Mechanism web page, Mechanical and Aerospace Engineering (Combustion Research), University of California at San Diego (http://combustion.ucsd.edu). 